DFTUZ/95/19

LPTHE Orsay- $95 / 50$

hep-lat/9507003

June 1995

(Revised version, September 1995)

\title{
A class of chiral fermion models
}

\author{
J.L. Alonso旬, Ph. Boucaud回, F. Lesmes 时 and \\ A.J. van der Sijs 2 \\ a Departamento de Física Teórica, Universidad de Zaragoza, 50009 Zaragoza, \\ Spain. \\ b Laboratoire de Physique Théorique et Hautes Energies, Université de Paris XI, \\ 91405 Orsay Cedex, France. 3
}

\begin{abstract}
We study the relation between the Roma and Zaragoza proposals for chiral fermions on the lattice. The fermion action in the Roma approach is shown to be equivalent to one of the Zaragoza type. This result is used to perform a mean-field study of the phase diagram for chiral Yukawa models based on the Roma action. The phase diagram is compared with the one based on the Zaragoza model with the most local choice for the fermion interactions.
\end{abstract}

\section{Introduction}

The formulation of a Chiral Gauge Theory (CGT) on the lattice suffers from the well-known doubling problem [四]. Several proposals to deal with this problem have been reviewed in Refs. [2, 3]. In some of them one tries to apply the recipe successfully used in vector-like gauge theories: a Wilson-like term is introduced in an attempt to give a large mass to the unwanted doublers. In the Smit-Swift model for example [4], a scalar field is introduced to write

1 Now at "Instituto de Magnetismo Aplicado Salvador Velayos", Apdo. de Correos 155, 28230 Madrid, Spain.

2 E-mail address: arjan@sol.unizar.es.

3 Laboratoire associé au CNRS. 
down a gauge invariant Wilson-Yukawa term. When the scalar field acquires a non-vanishing vacuum expectation value, a mass term is generated for all the fermions. In the perturbative regime in the continuum, however, a fermion with a mass created by a Yukawa interaction does not decouple when this mass becomes large [5,66,7]. The same phenomenon appears to occur on the lattice. The electroweak $S, U$ and $\Delta \rho$ parameters, for example, receive contributions from the doublers in the Smit-Swift model [8]. In the mirror fermion model [9], supplementary, interacting physical fermions (the mirror fermions) are introduced. They make the model vector-like, with chiral properties at low energy, so that a conventional gauge invariant Wilson term can be used; the doublers get a mass of the order of the cut-off and the parameters are tuned in such a way that the masses of the mirror fermions are sufficiently high to have remained unobserved so far. These additional heavy fermions still contribute to the above-mentioned electroweak parameters, though [8].

It seems that all models which try to produce heavy masses through the Higgs mechanism, to get rid of the unwanted fermions, are plagued by these non-decoupling effects. In the Zaragoza proposal [10,11, 12], the heavy fermions are avoided. Instead, the doublers are massless and non-interacting (see Sect. 2). In the Roma approach [13], referred to as Roma I in Ref. [14, auxiliary fermions are used but the decoupling of these additional particles is achieved through a symmetry property [15]. Both the Roma and Zaragoza approaches are gauge non-invariant regularizations. The Roma group has shown [13], however, that one can recover a gauge invariant theory by an (in general) nonperturbative tuning of counterterms. The Roma and Zaragoza methods both preserve the global chiral symmetry and it has been shown that they reproduce the correct values for the fermionic contributions to the electroweak parameters $S, U$ and (the "universal part" of) $\Delta \rho$ at one loop [8, 16]. The variant of the Roma model discussed in Ref. [17], known as Roma II [14, in which no redundant variables are used, and the Reduced Staggered Fermion Model of Smit and collaborators 18], should also correctly reproduce these parameters. However, as these models break the global chiral symmetry one would have to take into account the gauge non-invariant counterterms to achieve this [16]. Recently, a lot of effort has furthermore been dedicated to proposals related to domain-wall fermions and fermions in the continuum [19]. However, the computation of the electroweak parameters $S, U$ and $\Delta \rho$ using some implementation of these ideas has not yet been considered.

In this paper, 4 we shall establish a direct relation between the Roma I and Zaragoza actions. This relation is exploited to determine the phase diagram of chiral Yukawa models based on the Roma I approach using mean-field techniques. This phase diagram is compared with the one for the Zaragoza model with the most local fermion interaction.

$\overline{4}$ For a concise presentation of part of the work described here, see Ref. 20. 
The study of such phase diagrams is important in order to investigate where a continuum field theory may be sensibly defined. Clearly, one would like to recover (the gaugeless limit of) the Standard Model as a continuum limit of a lattice model. Furthermore, it is not excluded, that certain properties of quantum field theories not anticipated on the basis of perturbative methods, like non-trivial fixed points, will provide a hint how to "improve" or extend the Standard Model. The search for such hints is another incentive to study phase diagrams of chiral Yukawa models. In addition, the answer to questions such as: what is the maximal fermion mass which can be generated by a Yukawa coupling, what is the effect of a strong Yukawa coupling on the upper and lower bounds on the Higgs mass, etc., will depend on where in the phase diagram of the, say, $\mathrm{SU}(2) \times \mathrm{U}(1)$ chiral Yukawa model one approaches the continuum limit. Most of these questions have already been studied for the Smit-Swift model [21] and the mirror fermion model [22]. In particular, in the strong coupling symmetric phase of the former model, some perturbatively unexpected physics was found [23]. For a detailed discussion of possible continuum limits in general see the review of Ref. [24]. Some of these questions are currently also under investigation [25,26] for the model with the local Zaragoza form factor. Here, we will only briefly discuss some properties which can be analysed in the context of our mean-field computations, such as which particles survive in the continuum limit. We would like to recall, however, that in the Roma and Zaragoza formulations the Standard Model is defined in the scaling region on the broken side of the small- $y$ ferromagnetic-paramagnetic phase transition.

The remainder of this paper is laid out as follows. In Section 2, the main characteristics of the Zaragoza proposal are recalled. Section 3 reviews the auxiliary fermion method used in the Roma I action and establishes the equivalence with a model of the Zaragoza class. In Section 4 we calculate and compare phase diagrams of chiral Yukawa models in the mean-field approximation. Some subtle aspects of the Zaragoza phase diagram, extending the earlier work [27], are also discussed here. A summary and discussion can be

found in Section 5. Details of some of the calculations have been collected in Appendices A and B.

\section{The Zaragoza proposal}

The philosophy of the Zaragoza approach [10,11, 12] (see also Ref. [28]) to chiral fermions on the lattice is to tolerate the presence of the species doublers, but to prevent their communication with the real world: they are allowed to propagate but are kept massless and non-interacting. 
The kinetic term for the fermions is taken to be the naive one,

$$
\mathcal{S}_{\text {kin }}=\sum_{x, y} \bar{\psi}(x) K_{x, y} \psi(y)
$$

where $K$ is the usual lattice Dirac operator,

$$
K_{x, y}=\frac{1}{2} \sum_{\mu} \gamma_{\mu}\left(\delta_{y, x+\hat{\mu}}-\delta_{x, y+\hat{\mu}}\right)
$$

It takes care of the propagation of all $2^{d}$ fermion species.

The interaction terms are to be constructed in such a way that the doublers do not contribute. For this purpose, we introduce the quantity $\psi^{(1)}$ defined in momentum space by

$$
\psi^{(1)}(p)=F(p) \psi(p) .
$$

The "form factor" $F(p)$ is required to be 1 for $p=0$, and to vanish when $p$ equals any of the doubler momenta. The idea is to prevent $\psi^{(1)}$ from coupling to the doublers at tree level. For the function $F$, we usually choose (a different choice was taken in Ref. [11, for example)

$$
F(p)=\prod_{\mu} f\left(p_{\mu}\right), \quad f(\theta)=\cos \left(\frac{\theta}{2}\right), \quad \theta \in(-\pi, \pi],
$$

see Fig. 1. With this form factor (4), the relation between the fields $\psi^{(1)}$ and $\psi$ is the most local one in $x$-space: $\psi^{(1)}(x)$ sits in the center of the lattice hypercube at $x$ and is equal to the average of the $\psi$ fields living on the corners surrounding it. This is easy to implement in a numerical simulation.

The form factor $F$ picks out the fermion $\psi^{(1)}$ at $p=0$. Similarly, one can select the fermions $\psi^{(j)}$ centered around any of the doubler momenta $p_{1}=0$, $p_{2}=(\pi, 0, \ldots)$, etc., by defining

$$
\psi^{(j)}(p)=F^{(j)}(p) \psi(p), \quad F^{(j)}(p) \equiv F\left(p+p_{j}\right) \quad\left(j=1, . ., 2^{d}\right) .
$$

The particular form factor of Eq. (4) satisfies $\sum_{j}\left(F^{(j)}\right)^{2}=1$, so that the kinetic term $\bar{\psi} K \psi$ reduces to $\sum_{j} \bar{\psi}^{(j)} K \psi^{(j)}$. The fermionic measure in the path integral does not factorize, though.

The interaction terms are then formed in a straightforward way, using $\psi^{(1)}$ instead of $\psi$. For example, a lefthanded chiral gauge interaction is written as 


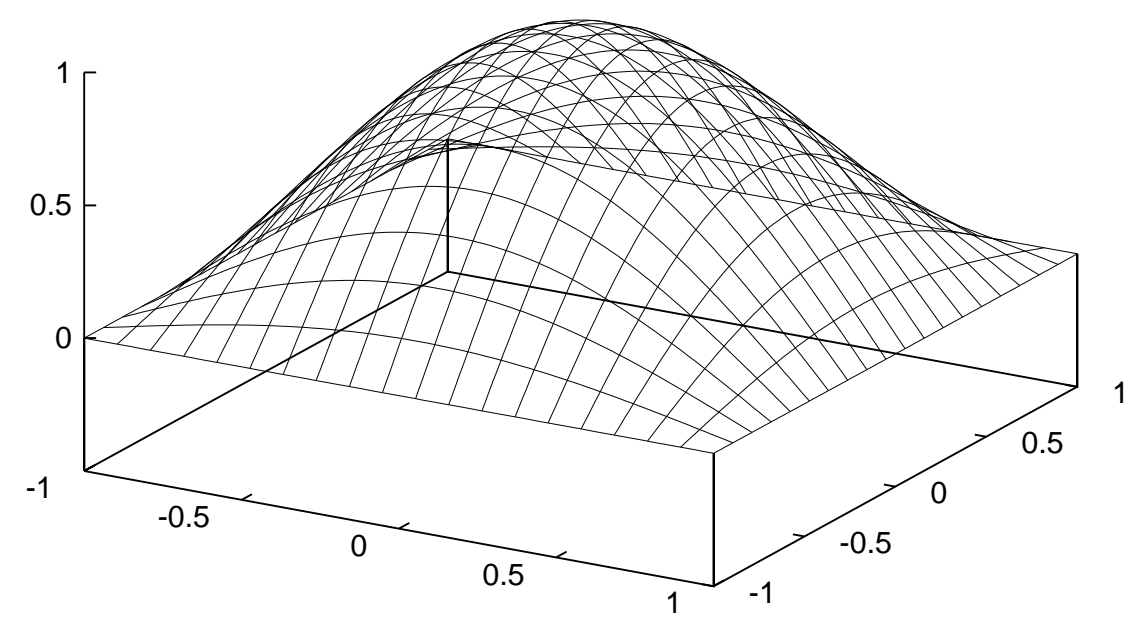

Form factor Roma

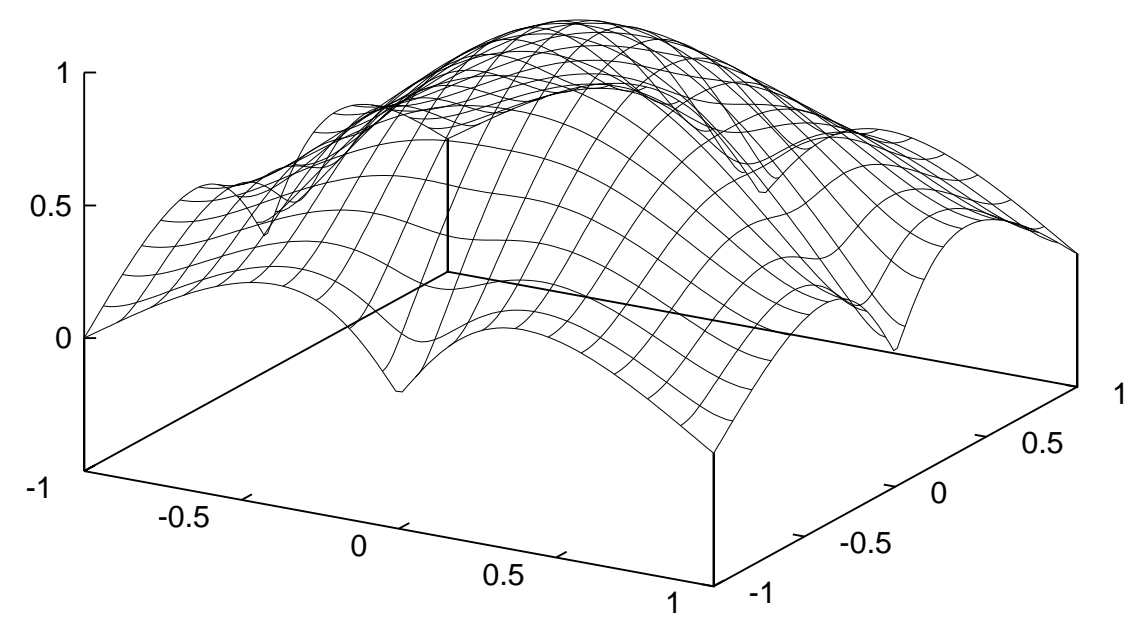

Fig. 1. Form factors $F\left(p_{1}, p_{2}\right)$ on the Brillouin zone in two dimensions (momenta in units of $\pi$ ), for the most local choice of Zaragoza form factor (四) (top) and for the Roma form factor (28), with parameter $r=1$ for the Wilson term (bottom). 


$$
\begin{array}{r}
\mathcal{S}_{U \psi}=\frac{1}{2} \sum_{x, \mu} \bar{\psi}_{L}^{(1)}(x) \gamma_{\mu}\left(U_{L \mu}(x)-1\right) \psi_{L}^{(1)}(x+\hat{\mu}) \\
-\bar{\psi}_{L}^{(1)}(x+\hat{\mu}) \gamma_{\mu}\left(U_{L \mu}^{+}(x)-1\right) \psi_{L}^{(1)}(x),
\end{array}
$$

where, as usual, $\psi_{L}^{(1)}=P_{L} \psi^{(1)}$ with $P_{L}=\frac{1}{2}\left(1-\gamma_{5}\right)$. Note that this interaction involves the lattice link variable $U_{L \mu}$ with the unit matrix subtracted. A Yukawa interaction between $\psi^{(1)}$ and a scalar field $\Phi$ will be of the form

$$
\mathcal{S}_{\Phi \psi}=y \sum_{x}\left(\bar{\psi}_{L}^{(1)}(x) \Phi(x) \psi_{R}^{(1)}(x)+\bar{\psi}_{R}^{(1)}(x) \Phi^{+}(x) \psi_{L}^{(1)}(x)\right) .
$$

More complicated interactions can be constructed as well. The key point is to use only $\psi^{(1)}$ as an interacting fermion field. In this way, all the vertices pick up form factors in momentum space which suppress the contributions coming from the doublers. One is left with only one physical, interacting fermion, while the doublers remain present but massless and decoupled.

This decoupling can be shown to hold beyond tree-level as well [12,28]. One approach is to use the Reisz power counting theorem on the lattice 229 (see also Ref. [30]), which formulates (sufficient) conditions for lattice Green's functions to tend to the corresponding continuum Green's functions (for undoubled fermions!) in the continuum limit. Anticipating a change of variables to be discussed shortly (18), we write the denominator of the propagator (19) as (temporarily restoring factors of the lattice spacing $a$ )

$$
D(a p)=s^{2} /\left(a^{2} F^{2}\right)+m^{2} F^{2},
$$

where we have defined

$$
s^{2}=\sum_{\mu} \sin ^{2} a p_{\mu}, \quad m=y\langle\Phi\rangle,
$$

and

$$
F^{2} \equiv F^{+} F
$$

(although we shall usually consider form factors which are real number valued functions). For application of Reisz' theorem, sufficient conditions are that this denominator is a $C^{\infty}$ function, tending to $p^{2}+m^{2}$ in the limit $a \rightarrow 0$, and satisfying

$$
D(a p) \geq A\left(\sum_{\mu} \frac{4}{a^{2}} \sin ^{2}\left(a p_{\mu} / 2\right)+m^{2}\right),
$$


on the entire Brillouin zone, for some constant $A$. (In addition, there are a few other, rather weak conditions on the numerators of the propagators and on the vertices which are satisfied in the present case and, in fact, in many other cases.) In the massless case, this inequality is easily verified for the local form factor $F(4)$ :

$$
s^{2} / a^{2} F^{2}=\frac{1}{a^{2}} \sum_{\mu} \frac{4 \sin ^{2}\left(a p_{\mu} / 2\right) \cos ^{2}\left(a p_{\mu} / 2\right)}{\prod_{\nu} \cos ^{2}\left(a p_{\nu} / 2\right)} \geq \sum_{\mu} \frac{4}{a^{2}} \sin ^{2}\left(a p_{\mu} / 2\right) .
$$

If $m \neq 0$, we need part of the $s^{2} /\left(2 a^{2} F^{2}\right)$ term in (8) to yield the $A m^{2}$ term in (11) near the edge of the Brillouin zone, where the factor $F^{2}$ multiplying the $m^{2}$-term becomes small. Writing, for example,

$$
D(a p)=s^{2} /\left(2 a^{2} F^{2}\right)+\left[s^{2} /\left(2 a^{2} F^{2}\right)+m^{2} F^{2}\right],
$$

the term between square brackets is larger than $2^{-d} m^{2}$ in the inner region where $p_{\mu} \leq \pi / 2$, and larger than $(1 / \mathrm{am})^{2} m^{2}$ in the rest of the Brillouin zone, and one readily finds a value of $A$ for which the inequality (11) is satisfied. The condition requiring $C^{\infty}$ behaviour of this denominator is not satisfied for this local form factor, but it can presumably be replaced by a somewhat weaker condition which would allow application of the theorem in this case. With a less local form factor, for instance the one following from the Roma action (see Sect. 3.1), the $C^{\infty}$ condition can also be satisfied.

In addition to this perturbative decoupling theorem, there are GoltermanPetcher shift symmetries [15] for the doublers [12,28]. In momentum space they take the form

$$
\begin{aligned}
& \psi(p) \longrightarrow \psi(p)+\epsilon \delta\left(p-p_{i}\right), \\
& \bar{\psi}(p) \longrightarrow \bar{\psi}(p)+\bar{\epsilon} \delta\left(p-p_{i}\right),
\end{aligned}
$$

where $\epsilon$ and $\bar{\epsilon}$ are independent parameters, $i=2, . ., 2^{d}$, and the $p_{i}$ are the doubler momenta. On the 'doubler fields' $\psi^{(j)}$ defined in Eq. (5), and their conjugates, these symmetry transformations $\left(i=2, . ., 2^{d}\right)$ act as

$$
\begin{aligned}
& \psi^{(j)}(p) \longrightarrow \psi^{(j)}(p)+\epsilon \delta_{i j} \delta\left(p-p_{j}\right), \\
& \bar{\psi}^{(j)}(p) \longrightarrow \bar{\psi}^{(j)}(p)+\bar{\epsilon} \delta_{i j} \delta\left(p-p_{j}\right),
\end{aligned}
$$

taking care of the decoupling of the doubler with label $i$ (for more details, see Ref. [28]). Clearly, the prospective physical fermion $\psi^{(1)}$ is not decoupled.

Evidently, the use of $\psi^{(1)}$ instead of $\psi$ in the interaction terms (6, ,7) does not respect the gauge invariance. As in the Roma I model [13], this means 
that gauge fixing and ghosts have to be introduced explicitly in the lattice definition of the model.' (We are not considering gauge fixing and ghost terms nor gauge-variant counterterms here because they are not relevant for our purposes.) A global chiral invariance is preserved, though, because $\psi^{(1)}(x)$ is a linear combination of $\psi$ fields at different sites. This global symmetry restricts the number of counterterms. For example, no fermion mass counterterms are needed.

As mentioned in the introduction, the Zaragoza method has been used to study the contribution of a doublet of fermions to the electroweak parameters $S, U$ and $\Delta \rho$ on the lattice. At one loop the continuum result was reproduced $[16 \rrbracket$, confirming the decoupling of the doublers. One should note that the gauge-variant counterterms, needed to recover the symmetries of the continuum, do not contribute to these parameters at the one-loop level. A lattice simulation aiming at a non-perturbative demonstration of the decoupling and a non-perturbative determination of the leading fermionic contribution to the universal part of $\Delta \rho$ is currently in progress [25,26].

It is interesting to note that a change of variables

$$
\psi(p) \rightarrow F^{-1}(p) \psi(p)
$$

transforms the original action (11,6, Ø), schematically written as

$$
\begin{aligned}
\mathcal{S} & =\bar{\psi} K \psi+\bar{\psi}^{(1)}(U-K) \psi^{(1)}+y \bar{\psi}^{(1)} \Phi \psi^{(1)} \\
& =\bar{\psi} K \psi+\bar{\psi} F^{+}(U-K) F \psi+y \bar{\psi} F^{+} \Phi F \psi
\end{aligned}
$$

into

$$
\mathcal{S}^{\prime}=\bar{\psi}\left(F^{+}\right)^{-1} K F^{-1} \psi+\bar{\psi}(U-K) \psi+y \bar{\psi} \Phi \psi
$$

where $U$ is the usual gauge covariant lattice Dirac operator. This transformation, with constant Jacobian, shifts the form factors from the vertices to the fermion propagator. In Feynman graphs, this can be visualized as follows. Every fermion-fermion-Higgs vertex from (17) contains a factor $F^{2} \equiv F^{+} F$ (and similarly for the interaction with the gauge field). These form factors can either be thought of as part of the vertex, as in (17), or be assigned to the fermion propagators connected to the vertex, as in (18). In fact, in the formulation of Eq. (18) the propagator (in the broken phase) has the form

$$
S(p)=\frac{-i \$+m F^{2}}{s^{2}+m^{2} F^{4}} F^{2}
$$

5 Ref. [31] describes a proposal for a practical implementation. 
where

$$
\oint=\sum_{\mu} \gamma_{\mu} \sin p_{\mu} .
$$

For $p \rightarrow 0, F \rightarrow 1$ and the propagator corresponds to a massive fermion. Due to the overall factor $F^{2}$, however, the poles at the doubler momenta are suppressed.

It is interesting to note that the "Zaragoza philosophy" mentioned at the beginning of this Section acquires a different interpretation in this formulation. While in the original form (17) species doublers were present, albeit massless and non-interacting, they are absent from the $\psi$-propagator following from Eq. (18). This is not in disagreement with the no-go theorem [1] because the action (18), in which the original $\psi^{(1)}$ is now the elementary fermion field, contains a non-local kinetic term.

\section{The Roma I approach}

One of the ideas to deal with the problem of chiral lattice fermions has been to introduce auxiliary righthanded partners $\chi_{R}$ for the lefthanded physical fields $\psi_{L}$. Then a Wilson term can be formed which is used to decouple the species doublers. Similarly, an auxiliary lefthanded partner $\chi_{L}$ is introduced for each of the physical righthanded fields $\psi_{R}$, with a corresponding Wilson term.

Ideas of this kind have been discussed in refs. [9, 13, 32, 33, with different uses.

The mirror fermion model [9], in which the auxiliary fermions are interacting fields, transforming under gauge transformations, is essentially vectorlike in nature; the auxiliary mirror fermions are physical. The idea is, however, to obtain an effective chiral theory at low energies by choosing the (both diagonal and off-diagonal) elements of the fermion mass matrix, or the matrix of Yukawa couplings to the Higgs field, in such a way that part of the spectrum resides at high enough energies.

If the auxiliary fields do not transform under gauge transformations, the Wilson term breaks the symmetry. In the work of Smit [32, 33], however, pursuing a gauge-invariant formulation, the Higgs fields evoked by acting with gauge transformations turn the Wilson terms into Wilson-Yukawa terms, thereby restoring the gauge invariance. 
In the Roma model [13], the auxiliary fields transform only under global transformations, so that the Wilson term breaks gauge invariance. The central idea of the Roma approach was, however, that the model has to be defined in the presence of gauge-fixing and ghost terms, so one must keep such terms also at the non-perturbative level. At the same time, one has to include all the counterterms needed to restore the BRST symmetry in the continuum limit. In this formulation, the Wilson term can be naturally maintained in its gauge non-invariant form.

We will discuss this gauge non-invariant form of the action with auxiliary fermions, and will refer to it as the Roma I action. In the absence of gauge fields, the mirror model action takes the form of the Roma action if all the relevant couplings to the mirror fields are set to zero (i.e., the bare pure and mixing mass parameters and the Yukawa coupling for the mirror fermions).

The fermion part of the Roma I action [13] consists of a minimally coupled kinetic term and a Yukawa interaction, supplemented with a Wilson term. It can be written in a condensed way as

$$
\begin{aligned}
\mathcal{S}_{\mathrm{R}}= & \bar{\psi}_{L} U_{L} \psi_{L}+\bar{\chi}_{R} K \chi_{R}+\bar{\psi}_{R} U_{R} \psi_{R}+\bar{\chi}_{L} K \chi_{L} \\
& +y \bar{\psi}_{L} \Phi \psi_{R}+y \bar{\psi}_{R} \Phi^{+} \psi_{L} \\
& +\bar{\psi}_{L} W \chi_{R}+\bar{\psi}_{R} W \chi_{L}+\bar{\chi}_{L} W \psi_{R}+\bar{\chi}_{R} W \psi_{L}
\end{aligned}
$$

The physical fermion fields are denoted by $\psi$, their auxiliary chiral partners by $\chi . W$ is a 'mixed' Wilson term (with parameter $r=1$ ),

$$
\bar{\psi} W \chi=\frac{1}{2} \sum_{x, \mu} \bar{\psi}(x)[\chi(x+\hat{\mu})+\chi(x-\hat{\mu})-2 \chi(x)],
$$

the rest of the notation is the same as in Sect. 2. We have not written the kinetic terms for the gauge, ghost and scalar fields, the scalar self-coupling, nor the gauge-variant counter-terms, because they are of no concern here.

This action is invariant under the global chiral transformations

$$
\begin{aligned}
& \psi_{L} \longrightarrow \Omega_{L} \psi_{L} \chi_{R} \longrightarrow \Omega_{L} \chi_{R} \\
& \bar{\psi}_{L} \longrightarrow \bar{\psi}_{L} \Omega_{L}^{+} \bar{\chi}_{R} \longrightarrow \bar{\chi}_{R} \Omega_{L}^{+} \\
& \psi_{R} \longrightarrow \Omega_{R} \psi_{R} \chi_{L} \longrightarrow \Omega_{R} \chi_{L} \\
& \bar{\psi}_{R} \longrightarrow \bar{\psi}_{R} \Omega_{R}^{+} \bar{\chi}_{L} \longrightarrow \bar{\chi}_{L} \Omega_{R}^{+} \\
& \Phi \longrightarrow \Omega_{L} \Phi \Omega_{R}^{+} \\
& U_{L \mu} \longrightarrow \Omega_{L} U_{L \mu} \Omega_{L}^{+} \\
& U_{R \mu} \longrightarrow \Omega_{R} U_{R \mu} \Omega_{R}^{+}
\end{aligned}
$$


All the terms in $\mathcal{S}_{\mathrm{R}}(21)$ containing auxiliary fields are left ungauged. This feature distinguishes it from the mirror fermion model [9]. As a consequence, the local chiral symmetry is broken. On the other hand, one gains an invariance under a constant shift of the $\chi$ fields,

$$
\begin{aligned}
\chi_{R}(x) & \longrightarrow \chi_{R}(x)+\eta_{R} \\
\chi_{L}(x) & \longrightarrow \chi_{L}(x)+\eta_{L}
\end{aligned}
$$

from which the decoupling of these fields follows [15].

\subsection{Roma à la Zaragoza}

Now we make the following observation. As the auxiliary fields appear at most quadratically in $\mathcal{S}_{\mathrm{R}}(21)$, it is possible to integrate them out exactly. This gives rise to an additional contribution

$$
-\bar{\psi}_{L} W K^{-1} W \psi_{L}-\bar{\psi}_{R} W K^{-1} W \psi_{R}
$$

to the kinetic term of the $\psi$ field, while the rest of the action remains unchanged. In the condensed notation of Eq. (21), the action becomes

$$
\begin{aligned}
\mathcal{S}_{\mathrm{R}}^{\prime}= & \bar{\psi}_{L} U_{L} \psi_{L}-\bar{\psi}_{L} W K^{-1} W \psi_{L} \\
& +\bar{\psi}_{R} U_{R} \psi_{R}-\bar{\psi}_{R} W K^{-1} W \psi_{R} \\
& +y \bar{\psi}_{L} \Phi \psi_{R}+y \bar{\psi}_{R} \Phi^{+} \psi_{L} \\
= & \bar{\psi}\left(F_{R}^{+}\right)^{-1} K F_{R}^{-1} \psi+\bar{\psi}(U-K) \psi \\
& +y \bar{\psi}\left(\Phi P_{R}+\Phi^{+} P_{L}\right) \psi
\end{aligned}
$$

if we take

$$
F_{R}=\sqrt{\frac{s^{2}}{s^{2}+w^{2}}}
$$

( $R$ for Roma), see Fig. 1, with $s^{2}=s^{2}(p)=\sum_{\mu} \sin ^{2} p_{\mu}$ and $w=w(p)=$ $\sum_{\mu}\left(1-\cos p_{\mu}\right)$.

The action (27) is exactly of the Zaragoza form (18), with a form factor given by (28).

Note that $F_{R}(p)$ satisfies the crucial form factor requirements $F_{R}(p=0)=1$ and $F_{R}\left(p=p_{i}\right)=0$ for the doubler momenta $p_{i}$. A difference between the

form factors (4) and (28) (cf. Fig. (1) is that $F_{R}=0$ only at the doubler 
momenta whereas the local form factor $F(\mathbb{\Psi})$ vanishes on the entire boundary hypersurface of the Brillouin zone. For small momenta $p$ both form factors go as $1-p^{2} / 8$, and in dimension $d=1$ they are identical. Note that, for $d \geq 2, F_{R}$ in Eq. (28) is not well-behaved at the doubler momenta $p_{i}$. It has conic behaviour, $F_{R}\left(p_{i}-k\right) \sim \sqrt{k^{2}}$, causing non-differentiability at these locations. This complicates an analytic study of the behaviour of $F_{R}$ in $x$ space, needed to determine the analogue of $\psi^{(1)}(x)$ for this case. Numerical calculations appear to indicate that $F_{R}(x=N a, y=0)$ and $F_{R}(x=y=N a)$ fall off like $(-1)^{N} / N^{3}$ in two dimensions.

It is interesting to have a look at the the fermion propagator given by the action (27). At tree level, in the broken phase, one reads off

$$
\begin{aligned}
S(p) & =\frac{-i \phi+m F_{R}^{2}}{s^{2}+w^{2}+m^{2} F_{R}^{2}} \\
& =\frac{-i \phi+m F_{R}^{2}}{s^{2}+m^{2} F_{R}^{4}} F_{R}^{2},
\end{aligned}
$$

where $m=y\langle\phi\rangle$. The Wilson term $w^{2}(p)$ in the denominator of Eq. (29) will decouple the doublers by the usual mechanism of giving them a mass of the order of the cutoff, while the conservation of global chiral symmetry is reflected by the absence of a $w$-term in the numerator. This is to be contrasted with the usual Wilson fermions where such a term, although formally of order $a$, gives a finite contribution due to its inclusion in divergent loops.

Alternatively, one may demonstrate the decoupling of the doublers by invoking the Reisz theorem or the Golterman-Petcher shift symmetry, as discussed in Sect. 2. The denominator of the fermion propagator is now a smooth function (i.e., it verifies the $C^{\infty}$-condition of Reisz' theorem), and in the symmetric phase the required inequality follows from

$$
\begin{aligned}
s^{2} / a^{2} F_{R}^{2} & =\left(s^{2}+w^{2}\right) / a^{2}=\frac{1}{a^{2}}\left\{\sum_{\mu} \sin ^{2} a p_{\mu}+\left(\sum_{\nu}\left[1-\cos a p_{\nu}\right]\right)^{2}\right\} \\
& =\frac{4}{a^{2}}\left\{\sum_{\mu} \sin ^{2}\left(a p_{\mu} / 2\right) \cos ^{2}\left(a p_{\mu} / 2\right)+\left(\sum_{\nu} \sin ^{2}\left(a p_{\nu} / 2\right)\right)^{2}\right\} \\
& \geq \frac{4}{a^{2}} \sum_{\mu} \sin ^{2}\left(a p_{\mu} / 2\right) .
\end{aligned}
$$

In the broken phase, near the edge of the Brillouin zone, part of the $w^{2}$-term in the denominator of the propagator (29) has to provide the mass term, as in the model with the local Zaragoza form factor, cf. Eq. (13). In this outer region, where at least one of the momentum components is large, $\left|p_{\nu}\right| \geq \pi / 2$, we have $w^{2} \geq 1$ and the inequality follows easily. 
The applicability of the GP shift symmetry is independent of the choice of form factor $F$, so we simply refer to the earlier remarks (14 15) for the decoupling of the doublers in Roma 'à la Zaragoza'.

\section{Phase diagrams of Chiral Yukawa Models}

The equivalence of the Roma I action to a Zaragoza type of action can be exploited to study the phase diagram of chiral Yukawa models based on the Roma I action.

The phase diagram for an $\mathrm{SU}(2)_{L} \times \mathrm{SU}(2)_{R}$ Chiral Yukawa model in the Zaragoza approach with the local form factor $F$ (四) was studied in Ref. [27, using both mean-field techniques and lattice simulations for $n_{f}=2$ fermion doublets. The mean field calculation, used in combination with a small or large- $y$ expansion, was shown to give a rather good approximation to the Monte Carlo results as far as the structure of the phase diagram is concerned.

Here we shall study the phase diagram of the corresponding chiral Yukawa model with the Roma I action using the mean-field techniques of Ref. [27]. Some details of this approach, which combines more standard mean-field techniques with expansions in the Yukawa coupling $y$, are described in Appendix A. We shall also present some new results for the Zaragoza phase diagram with the most local form factor, extending the previous analysis [27]. The results of the mean-field calculations for both phase diagrams are plotted in Fig. 2.

The action for $n_{f}$ degenerate fermion doublets is given by

$$
\begin{aligned}
\mathcal{S}= & -\frac{k}{2} \sum_{x, \mu} \operatorname{Tr}\left(\Phi^{+}(x) \Phi(x+\hat{\mu})+\Phi^{+}(x+\hat{\mu}) \Phi(x)\right) \\
& +\sum_{i=1}^{n_{f}}\left[\sum_{x, \mu} \frac{1}{2}\left(\bar{\psi}_{i}(x) \gamma_{\mu} \psi_{i}(x+\hat{\mu})-\bar{\psi}_{i}(x+\hat{\mu}) \gamma_{\mu} \psi_{i}(x)\right)\right. \\
& \left.+\sum_{x} y \bar{\psi}_{i}^{(1)}(x)\left(\Phi(x) P_{R}+\Phi^{+}(x) P_{L}\right) \psi_{i}^{(1)}(x)\right]
\end{aligned}
$$

for arbitrary choice of the form factor $F$ used in the definition of the interacting fermion field $\psi^{(1)}$. The modulus of the Higgs field is frozen. This corresponds to infinite bare Higgs self-coupling $\lambda$. With triviality in mind, we do not expect this to be a severe restriction. The phase diagram is given in terms of the two parameters $y$ and $k$. Because of the symmetry $\Phi \rightarrow-\Phi, y \rightarrow-y$, the $y$ values can be restricted to the range $y \geq 0$. For $y=0$ we have the $\mathrm{O}(4)$ model with critical points $\pm k_{c}$. The same is true for $y=\infty$, as can be seen after rescaling the fermion fields, $\psi \rightarrow \psi / \sqrt{y}$. There are some subtle aspects to the $y \rightarrow \infty$ 


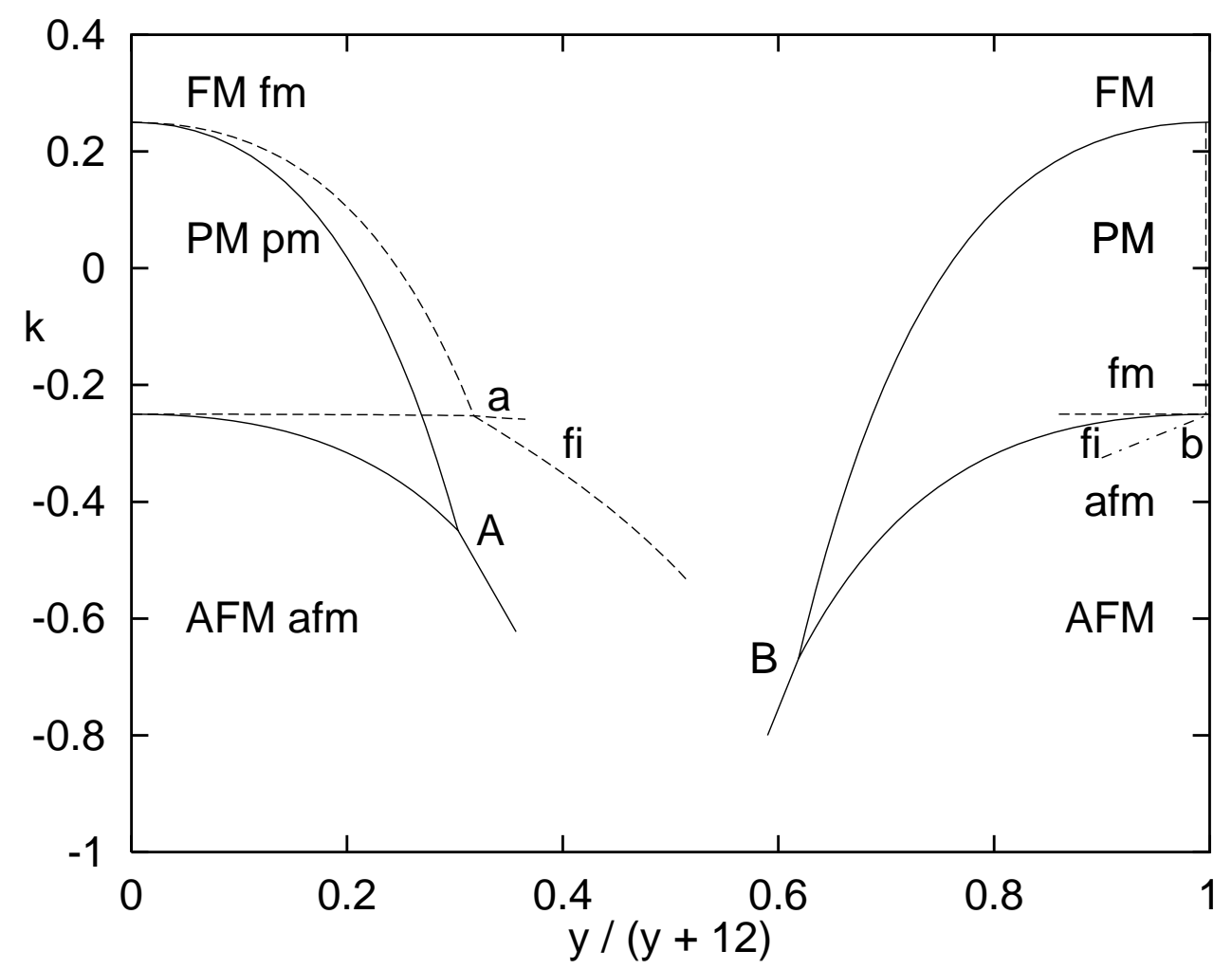

Fig. 2. Mean-field phase diagram for $\mathrm{SU}(2) \times \mathrm{SU}(2)$ chiral Yukawa models with 2 fermion doublets, for the local Zaragoza form factor (dashed curves) and for the Roma form factor (solid curves). Phases and multicritical points are indicated with capitals for the Roma case and with lowercase letters for the local Zaragoza case. The mean field result for the large- $y$ FM-FI transition in the local case, which is reliable only for $y \gtrsim 10^{7}$ and would be invisible for the present choice of scale along the horizontal axis, has been extended to smaller $y$ for clarity. The exact (mean-field) location of the FI-AFM transition in the region of strong Yukawa coupling, for the local form factor (dash-dotted line), is unknown.

limit in the case of the local form factor, though; we will come back to this later.

In the region of weak Yukawa coupling $y$, one finds paramagnetic (PM), ferromagnetic (FM) and antiferromagnetic (AFM) phases separated by the second order transition lines (cf. Appendix A)

$$
\begin{aligned}
& k_{\mathrm{pf}}^{(W)}(y)=\frac{1}{4}-y^{2} \frac{n_{f}}{2} I_{\mathrm{pf}}^{(W)}, \\
& k_{\mathrm{pa}}^{(W)}(y)=-\frac{1}{4}-y^{2} \frac{n_{f}}{2} I_{\mathrm{pa}}^{(W)},
\end{aligned}
$$

where 


$$
\begin{aligned}
& I_{\mathrm{pf}}^{(W)}=\int_{-\pi}^{\pi} \frac{\mathrm{d}^{4} p}{(2 \pi)^{4}} \frac{F^{4}(p)}{s^{2}(p)}, \\
& I_{\mathrm{pa}}^{(W)}=\int_{-\pi}^{\pi} \frac{\mathrm{d}^{4} p}{(2 \pi)^{4}} \frac{F^{2}(p) F_{\pi}^{2}(p)}{s^{2}(p)},
\end{aligned}
$$

with $F_{\pi}(p) \equiv F\left(p_{1}+\pi, \cdots, p_{4}+\pi\right)$. These curves meet in a point $A$ with coordinates

$$
y_{A}=1 / \sqrt{n_{f} I_{-}^{(W)}}, \quad k_{A}=-I_{+}^{(W)} / 4 I_{-}^{(W)},
$$

where

$$
I_{ \pm}^{(W)}=I_{\mathrm{pf}}^{(W)} \pm I_{\mathrm{pa}}^{(W)}
$$

For the local Zaragoza form factor $F$ one finds

$$
I_{\mathrm{pf}}^{(W)}=1.617910^{-2}, \quad I_{\mathrm{pa}}^{(W)}=8.412410^{-5},
$$

hence

$$
y_{A}=7.8823 / \sqrt{n_{f}}, \quad k_{A}=-0.25261
$$

while for the Roma form factor $F_{R}$

$$
I_{\mathrm{pf}}^{(W)}=2.570310^{-2}, \quad I_{\mathrm{pa}}^{(W)}=7.334310^{-3},
$$

and

$$
y_{A}=7.3783 / \sqrt{n_{f}}, \quad k_{A}=-0.44964 .
$$

One sees that the local form factor $F$ suppresses the contribution from the regions near the doubler momenta stronger than $F_{R}$ does: $I_{\text {pa }}^{(W)}$ is very small for the local case (39) and the transition line between the PM and AFM phases is almost horizontal.

As mentioned in Sect. 3, in the absence of gauge fields the mirror fermion action takes the form of the Roma action if all the relevant couplings to the mirrors are set to zero. In Ref. [22], the mirror fermion model with $\mathrm{SU}(2) \times \mathrm{SU}(2)$

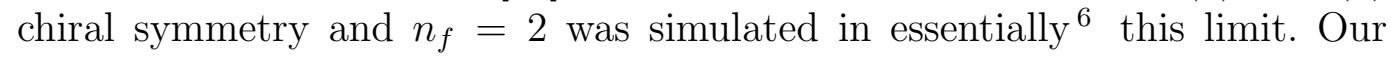
${ }^{6}$ A minor difference is that the simulation of Ref. [22] was carried out for a small non-zero bare mixing mass $\mu_{\psi \chi}=1-8 K=\mathcal{O}\left(1 / N_{t}^{2}\right)$. 
mean-field results agree quite well with Fig. 1. of Ref. [22], showing the small$y$ region of the phase diagram, as can be seen after the corresponding mapping of parameters $\left(y \rightarrow G_{\psi} / 2 K\right.$ with $\left.K \approx 0.125\right)$.

Beyond the point $A$, one may consider the possibility of a ferrimagnetic (FI) phase, characterized by nonzero expectation values for the fields as well as the staggered fields (in the staggered average, fields at the odd sites contribute with a minus sign). The mean field method can also be applied to this situation [27], and the equations for the transition lines can be solved numerically. The reliability of the $y$-expansion involved, in this intermediate- $y$ region, is monitored in terms of the quantity $\left|y \sum_{i=1}^{n_{f}}\left\langle\bar{\psi}_{i}^{(1)} \psi_{i}^{(1)}\right\rangle\right|$. In addition, one must check whether the free energy of the FI solution is actually lower than that of the competing FM and AFM solutions.

For the local form factor, a FI phase was found [27], separated from the FM and AFM phases by second order phase transition lines. In Fig. 2, these lines have been plotted up to the point where $\left|y \sum_{i=1}^{n_{f}}\left\langle\bar{\psi}_{i}^{(1)} \psi_{i}^{(1)}\right\rangle\right|=1$. Comparison with data from the lattice Monte Carlo simulation of the model showed that the presence and location of the FI phase are predicted quite well by the mean field results [27].

We have used the same numerical mean field methods to look for a FI phase in the model with the Roma form factor. In this case, however, the assumption of second order phase transitions between FM and FI phases, and between AFM and FI phases, led to the inconsistent result of a FM-FI transition line lying below the AFM-FI line.

In order to discover which of these candidate lines is (are) not acceptable, we made contour plots of the free energy, see Fig. 3. Curves of constant free energy $\mathcal{F}<0$ were determined for FM and AFM candidate solutions. The FM contour curves run roughly parallel to the PM-FM transition and its continuation, where $\mathcal{F}=0$, and similarly the AFM curves parallel to the PMAFM transition and its continuation. Subsequently, we determined the line of intersection points, at which the FM and AFM solutions have equal free energy. This line was found to lie in between the mutually inconsistent second order lines, implying that neither of the second order lines is acceptable: Above the line of intersection points of the free energy curves, no AFM solution is possible because the FM solution in the same point has lower free energy. Thus the AFM-FI line, found to lie above this intersection line, cannot be a transition between an AFM and a FI phase. Similarly, the FM-FI line is unacceptable. This is not yet sufficient to exclude a FI phase beginning at $A$. A FI phase bounded by first order transitions would still be possible. However, further inspection of the free energies for FM, AFM and FI mean field solutions in this region, using similar methods, confirmed that a FI phase is absent (at least close to the point $A$ ), while the FM and AFM phases are joined by a first 


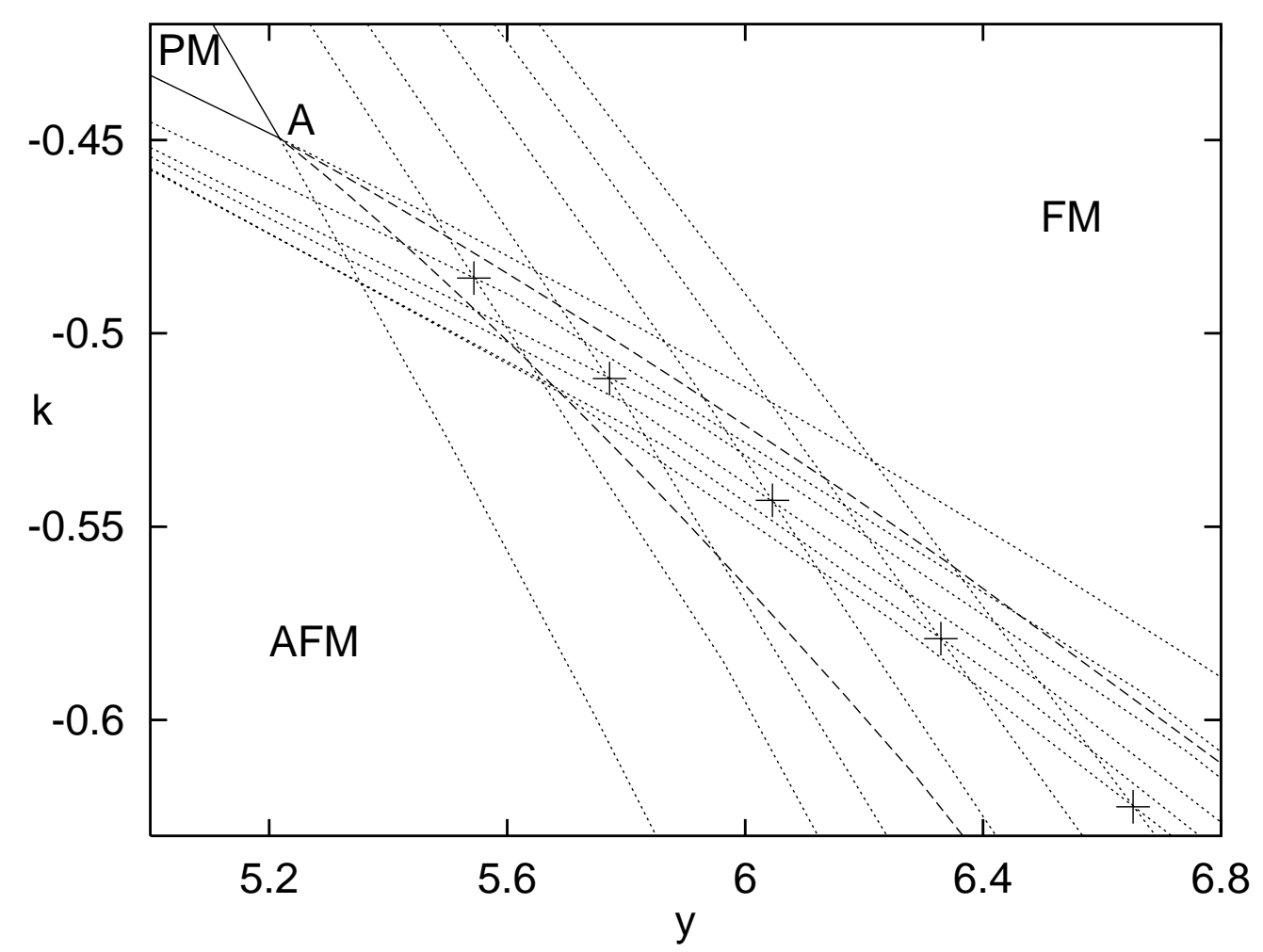

Fig. 3. Contour plot of the free energy for FM and AFM solutions in the region just beyond the weak-coupling PM phase. The solid lines are the mean-field results for the second order transitions between the PM phase, where the free energy is zero, and the FM and AFM phases. The mutually inconsistent (inverted) results for second order FM-FI and AFM-FI transitions are drawn as dashed lines. The dotted curves connect points at which FM or AFM solutions have a given free energy. The predominantly vertical curves are those for the FM solutions, with free energies $0=f_{0}>f_{1}>\ldots>f_{5}$, decreasing from left to right. The predominantly horizontal curves correspond to the AFM solutions, with the same values $f_{0, \ldots, 5}$ for the free energy, now decreasing from top to bottom. Points at which the FM and AFM solutions have equal free energy are indicated with the symbol + .

order transition. This transition line is determined by the line of intersection points in Fig. 3. It remains possible, though, that this first order line has an end point with a FI phase opening up beyond it [34].

At this point it is appropriate to make a remark about the coexistence of different phases at the point $A$. It was stated in Ref. [35] that, in the mean-field approximation, the FM-PM transition line should continue beyond the point $A$ as an AFM-FI transition, with the same slope, and similarly the AFM-PM line would turn into a FM-FI transition line with the same slope at $A$. The existence of the point $A$ would thus automatically imply the presence of a FI phase. This is not correct, as was shown in Ref. 34]. In fact, the FI phase may be absent, as in the model with the Roma form factor studied here, and if it is present then all four slopes at the point $A$ 
may be different, as in the model with the local Zaragoza form factor. The validity of these arguments does not depend on the details of the mean-field approximation, such as the expansion in $y$ used to deal with the fermion determinant. Quantitatively, however, different values for the slopes will be found for different approximations to the fermion determinant. In particular, the conclusion that a FI phase is absent in the model with the Roma form factor might have to be revised. Apart from this, one should of course keep in mind the limitations of the mean-field approximation. Fig. 1. of Ref. [22], reporting the earlier-mentioned Monte Carlo study of the mirror model in the limit that it is essentially equivalent to the Roma action, displays a FI phase beyond the point $A$, but the authors remark that this region was not investigated in detail.

Similarly, the phase structure in the large- $y$ region can be studied by combining mean-field with a 1/y expansion [27] (see also Appendix A). One expects PM, FM and AFM phases, as in the $\mathrm{O}(4)$ model to which the model reduces for $y \rightarrow \infty$. Assuming second order transitions between such phases, one finds transition lines given by the curves

$$
\begin{aligned}
& k_{\mathrm{pf}}^{(S)}(y)=\frac{1}{4}-\frac{1}{y^{2}} \frac{n_{f}}{2} I_{\mathrm{pf}}^{(S)}, \\
& k_{\mathrm{pa}}^{(S)}(y)=-\frac{1}{4}-\frac{1}{y^{2}} \frac{n_{f}}{2} I_{\mathrm{pa}}^{(S)},
\end{aligned}
$$

where

$$
\begin{aligned}
& I_{\mathrm{pf}}^{(S)}=\int_{-\pi}^{\pi} \frac{\mathrm{d}^{4} p}{(2 \pi)^{4}} \frac{s^{2}(p)}{F^{4}(p)}, \\
& I_{\mathrm{pa}}^{(S)}=\int_{-\pi}^{\pi} \frac{\mathrm{d}^{4} p}{(2 \pi)^{4}} \frac{s^{2}(p)}{F^{2}(p) F_{\pi}^{2}(p)} .
\end{aligned}
$$

These lines meet in a point $B$ parametrized by

$$
y_{B}=\sqrt{n_{f} I_{-}^{(S)}}, \quad k_{B}=-I_{+}^{(S)} / 4 I_{-}^{(S)},
$$

with

$$
I_{ \pm}^{(S)}=I_{\mathrm{pf}}^{(S)} \pm I_{\mathrm{pa}}^{(S)}
$$

The integrands of $I_{\mathrm{pf}, \mathrm{pa}}^{(S)}(42)$ are just the inverse of the integrands of $I_{\mathrm{pf}, \mathrm{pa}}^{(W)}$ (34). Is is easy to show, however, that for any form factor for which the integrals converge, both $I_{-}^{(W)}>0$ and $I_{-}^{(S)}>0$, so that the points $A$ and $B$ are 
guaranteed to exist. (Here we have an amusing example of positive functions $f, g$ satisfying both $\int f>\int g$ and $\int 1 / f>\int 1 / g$.)

For the Roma form factor (28), one finds

$$
I_{\mathrm{pf}}^{(S)}=348.01, \quad I_{\mathrm{pa}}^{(S)}=158.70,
$$

hence

$$
y_{B}=13.759 \sqrt{n_{f}}, \quad k_{B}=-0.66917 .
$$

As before, we also looked for a FI phase in the intermediate- $y$ region by solving the corresponding mean field equations numerically. The result turned out to be analogous to that for the small-y equations: An anomalous inversion between the assumed second order FM-FI and AFM-FI transition lines, with free energy considerations deciding in favour of FM and AFM phases separated by a first order transition. Again, the mean-field scenario does not exclude the possibility that a FI phase opens up some distance away from the point $B$, while the conclusion about the absence of a FI phase at $B$ may again be modified by an improved treatment of the fermion determinant.

For the local Zaragoza form factor the integrals $I_{\mathrm{pf}, \mathrm{pa}}^{(S)}$ 42) are divergent, implying the absence of a PM phase at large (finite) $y$ in this approximation. FM and FI phases were found, though [27], separated by an almost horizontal transition line. The 1/y-expansion involved in this case was estimated to be reliable for extremely large $y$ values only, $y \gtrsim 10^{7}$.

The absence of a PM phase is reminiscent of the model of Ref. [36]. There, the fermions at a site $x$ are coupled to the Higgs fields at the vertices of the hypercube at $x$. In the present model, the Higgs field at a site $x$ is coupled to fermions at the vertices of the hypercube at $x$, so one might be tempted by the similarity to assign this model to the same class. In the model of Ref. [36], however, there is no equivalence to an $\mathrm{O}(4)$ model in the $y \rightarrow \infty$ limit, whereas in the Zaragoza model with the local form factor such an equivalence is established by rescaling the fermion fields, $\psi^{(1)} \rightarrow \psi^{(1)} / \sqrt{y}$, after which the fermions decouple. On the basis of this equivalence one can argue that the present model should be of the "funnel" type [37], with FM, PM and AFM phases in the large- $y$ region.

The resolution to this paradox is somewhat subtle but quite interesting. Details of the calculations, in mean field, are given in Appendix B. It turns out that the phase diagram of the model with the local form factor can be considered as a limiting case of a funnel-shaped diagram, in which the PM phase has been pushed towards $y=\infty$. It has not disappeared, but is contracted onto 
the line $y=\infty$. The line element $\left(y=\infty,-k_{c}<k<k_{c}\right)$ is a second order phase transition line between the FM phase at large but finite $y$ and the PM phase at $y=\infty$, and the multicritical point $B$ coincides with the critical point $-k_{c}$ of the $\mathrm{O}(4)$ model. In particular, the values of the mean fields are found to decay with critical exponents (calculated explicitly in Appendix B), when this line element is approached along lines of constant $k$. It would be interesting to study this region, in particular the vicinity of the point $\left(y=\infty, k=k_{c}\right)$, by numerical simulation on the lattice.

An interesting question is whether the AFM phase of the $\mathrm{O}(4)$ model at $y=\infty$ extends into the finite- $y$ region. The same numerical approach mentioned earlier is applicable in principle to investigate this. In practice there is a technical difficulty, however. The method makes use of the values of certain integrals which diverge in the limit $y \rightarrow \infty$. At the extremely large $y$ values required by the reliability condition these integrals are very hard to evaluate. We have therefore not been able to establish the precise location of the AFMFI transition with these methods, but there are indications that the transition exists (the dash-dotted line in Fig. 2), with the AFM phase extending into the finite- $y$ region.

In conclusion, we have found that the $\mathrm{SU}(2) \times \mathrm{SU}(2)$ chiral Yukawa model (32) with Zaragoza fermions generically has a phase diagram of the funnel type. An example is obtained by taking the form factor (28) describing the Roma action. If the form factor is chosen to be the most local one (田), we get the limiting case in which the large- $y$ PM phase is pushed towards the line $y=\infty$. Alternative local form factors, for which the integrals (42) are also divergent, show similar behaviour.

In general, the dependence of the phase transition lines on the form factor can be sketched as follows. If the form factor $F$ suppresses the doublers strongly, i.e., if $F$ is relatively small in the vicinity of the doubler momenta, then the integral $I_{\mathrm{pa}}^{(W)}$ (34) is very small. The small-y PM-AFM transition line will then be almost horizontal. The integral $I_{\mathrm{pf}}^{(W)}(34)$, which receives its dominant contribution from momenta around the origin, will be affected only weakly, but still the small- $y$ PM-FM transition will be curved relatively little in this case. The PM-FM and PM-AFM transitions in the strong coupling region, on the other hand, are determined by the integrals $I_{\mathrm{pf}, \mathrm{pa}}^{(S)}(42)$, whose integrands are the inverses of those of $I_{\mathrm{pf}, \mathrm{pa}}^{(W)}$. These transition lines will thus be curved relatively strongly. As a general trend, modifying the form factor causes the transition lines at weak coupling to bend downwards and those at strong coupling to turn upwards, or vice versa. 


\section{Discussion}

We have established a relation between the Roma I and Zaragoza methods to put chiral fermions on the lattice. Both proposals preserve the global chiral symmetry and have been shown to reproduce, at one loop, the expected values of the leading fermionic contribution to the electroweak parameters $S$, $U$ and $\Delta \rho$. The decoupling of the fermion doublers and the protection against mass counter-terms due to the global chiral symmetry make these approaches well-suited for a non perturbative study of Chiral Yukawa Theories and in particular of the scalar-fermion sector of the Standard Model. One can, for example, perform a lattice Monte Carlo study of the decoupling of the doublers, and also a non-perturbative lattice computation of the most significant contributions to $\Delta \rho$ is possible within the fermion-scalar sector of the Standard Model 25,26.

We have demonstrated that the action in the Roma I formulation (where auxiliary fermions are present) can be rewritten 'à la Zaragoza' with the form factor given in Eq. (28). In $x$-space, this form factor is spread out widely, contrary to the most local possible choice usually made for the Zaragoza method (Eqs. (3-4)) which is easy to implement in numerical simulations.

An interesting question would be whether other globally chirally invariant fermion actions can be rewritten in the Zaragoza formulation, with some particular form factor.

The reformulation of the Roma I action as a Zaragoza model was exploited to calculate the phase diagram of chiral Yukawa models based on the Roma I action in the mean-field approximation (combined with expansions in the Yukawa coupling $y$ or $1 / y$ ). The results agree quite well with the Monte Carlo simulations in the small- $y$ region of Ref. 222]. In general, we argued that (meanfield) phase diagrams of chiral Yukawa models with Zaragoza fermions are of the "funnel" type, with PM, FM and AFM phases in both the weak and strong Yukawa coupling regions. For a class of local form factors, for which the fermion interactions are within the range of a few lattice sites, our meanfield calculation suggests that the PM phase in the large- $y$ region is contracted onto the $y=\infty$ axis, part of which is at the same time a phase transition line. One should keep in mind, however, that higher order corrections in the $1 / d$ expansion might change this conclusion.

Of course, in the scaling region the physical quantities are expected to be the same for any form factor which kills the doublers. In particular, the physics at the FM side of the strong FM-PM phase transition in the Roma case should be the same as in the model with the local form factor. As remarked in Appendices $\mathrm{A}$ and $\mathrm{B}$, at strong Yukawa coupling $y$ the fermions do not 
survive the continuum limit, a similar conclusion as was drawn in the SmitSwift model. In the small-y scaling region, perturbation theory and mean field indicate that the fermion content is as expected, with only the physical chiral fermion surviving in the continuum limit.

Let us conclude with a brief speculation about a different region in the phase diagram where one might contemplate taking a continuum limit, namely the scaling region on the AFM side of the AFM-PM phase transition. Although the AFM and FI phases could be lattice artefacts (absent for certain non-cubic lattice geometries), there appears to be no rigourous argument precluding the existence of a (possibly non-trivial) continuum limit at an AFM-PM phase transition [38]. If such a limit makes sense, it may be interesting to investigate, for example, a weak-coupling scenario with fermions which are naturally light on the scale of $246 \mathrm{GeV}$ : the fermions, with a mass proportional to $v$ in leading order would remain (almost) massless in, say, a mean-field approach, while the gauge boson mass squared, proportional to $k\left\langle\phi_{x}^{+} \phi_{x+\hat{\mu}}\right\rangle$, would presumably not differ appreciably from its value in the weak FM scaling region.

\section{Acknowledgements}

This work was supported by EC contracts CHRX-CT92-0051 and ERBCHBICT941067, by DGICYT Spain, project AEN 94-218, by Acción Integrada Hispano-Francesa HF94-150B and by the National Science Foundation under Grant No. PHY94-07194.

\section{A Appendix}

Here we present some details of the mean field computation. The most significant characteristics of the approach have been described in Ref. [27]. As our starting point we shall take Eq. 11 of that reference, giving the mean field free energy for the $\mathrm{SU}(2) \times \mathrm{SU}(2)$ chiral Yukawa model of Eq. (32),

$$
\begin{array}{r}
\mathcal{F}=-\frac{1}{N} \log Z=\frac{1}{2}\left(\lambda^{2}+\bar{\lambda}^{2}\right)-2 d k\left(v^{2}-\bar{v}^{2}\right)+\alpha v+\bar{\alpha} \bar{v} \\
-\frac{1}{2}[u(\alpha+\bar{\alpha})+u(\alpha-\bar{\alpha})]-C_{0} I
\end{array}
$$

Here $C_{0}=2^{[d / 2]} n_{f} / 2, n_{f}$ is the number of fermion doublets, $d$ is the dimension of space-time, and $u(\alpha)=\log \left(2 I_{1}(\alpha) / \alpha\right)=\alpha^{2} / 8-\alpha^{4} / 384+\mathcal{O}\left(\alpha^{6}\right)$ for $\mathrm{SU}(2)$, with $I_{1}$ the modified Bessel function of order $1 . \mathcal{F}$ is a function of the mean fields $\alpha, v$ and $\lambda$, collectively denoted as $h_{i}$, and of the staggered mean fields $\bar{\alpha}, \bar{v}$ and $\bar{\lambda}$, denoted as $\bar{h}_{j}$, and is parametrized by the hopping parameter $k$ 
for the Higgs field and the Yukawa coupling $y$. The quantities $\alpha$ and $v$, and $\bar{\alpha}$ and $\bar{v}$, are mean field values for auxiliary fields needed to describe the scalar field, and $\lambda$ and $\bar{\lambda}$ are related to an auxiliary field corresponding to the fermion condensate $\left\langle\bar{\psi}^{(1)} \psi^{(1)}\right\rangle$ (see Ref. [27] for details).

The function $I$ comes from the fermionic determinant. It is obtained after a truncation of terms of higher order than quadratic in $y($ or $1 / y)$ in the mean field average of the Yukawa terms. In fact, the real expansion parameter here is $y n_{f}\left\langle\bar{\psi}^{(1)} \psi^{(1)}\right\rangle$ rather than $y$ (and $y^{-1} n_{f}\left\langle\bar{\eta}^{(1)} \eta^{(1)}\right\rangle$ rather than $y^{-1}$ in the large- $y$ region, where $\eta$ and $\bar{\eta}$ are auxiliary fermion fields [27]), so that this approximation should be valid in and near the paramagnetic phases [27]. For illustration we shall consider the region of weak Yukawa coupling, where $I$ has the form

$$
I=\int_{-\pi}^{\pi} \frac{d^{d} p}{(2 \pi)^{d}} \log \frac{\left[s^{2}(p)+\left(m^{2}-\bar{m}^{2}\right) F^{2}(p) F_{\pi}^{2}(p)\right]^{2}+m^{2} s^{2}(p)\left[F^{2}(p)-F_{\pi}^{2}(p)\right]^{2}}{s^{4}(p)}
$$

Here $F(p)$ is the form factor, $F_{\pi}(p)=F\left(p_{1}+\pi, \ldots, p_{d}+\pi\right), s^{2}(p)=\sum_{\mu} \sin ^{2}\left(p_{\mu}\right)$, and $m=\left(m_{+}+m_{-}\right) / 2, \bar{m}=\left(m_{+}-m_{-}\right) / 2$ with

$$
m_{ \pm}=y\{\dot{u}(\alpha \pm \bar{\alpha})-(\lambda \pm \bar{\lambda}) \sqrt{\ddot{u}(\alpha \pm \bar{\alpha})}\}
$$

The dots on the function $u(\alpha)$ denote derivatives. We have normalized Eq. (A.2) such that the free energy of the PM phase is zero. Concerning the mass $m_{f}$ (in lattice units) of the physical fermions in the scaling region of the FM phase, we can, as usual, distinguish a weak and a strong FM region. In fact, it can be shown from Eqs. (A.1, A.2) that $m_{f}^{2}=m_{+}^{2}\left(=y^{2} z^{2}\right.$ in the notation of Ref. [27]) in the small- $y$ FM phase. For large $y$ the result is $m_{f}^{2}=y^{2} / z^{2}$ [27].

To find the phase of the system for given $y$ and $k$, the free energy is minimized with respect to the mean fields (for a recent concise discussion see Ref. [34]), for which a necessary condition is that the saddle-point equations

$$
\frac{\partial \mathcal{F}}{\partial h_{i}}=\frac{\partial \mathcal{F}}{\partial \bar{h}_{j}}=0
$$

are satisfied. A second order phase transition from a symmetric to a broken phase occurs when a negative mode develops and the mean fields acquire nonzero values. In general, such a transition is given by the condition

$$
\operatorname{det} \mathcal{F}^{\prime \prime}(h, \bar{h})=0 \text {. }
$$

This equation is hard to solve for the case that there are broken phases on 
both sides of the transition under consideration (for example, a transition between antiferromagnetic (AM) and ferrimagnetic (FI) phases). Then Eq. (A.5) has to be satisfied for the non-trivial mean field values given by Eq. (A.4). If one wants to solve these equations perturbatively by expanding $\mathcal{F}$ in powers of the mean fields, then it is crucial [34] to include terms up to at least fourth order in the fields. Alternatively, a numerical approach may be adopted to find the exact solution, as in Ref. [27] and in the present paper. It should be emphasized, however, that the assumption of second order phase transition lines should be checked afterwards by comparing free energies of different mean field values.

If either side of the transition is PM, however, then Eq. (A.5) is taken at zero mean fields and can be solved easily. One can for example expand $\mathcal{F}$ to quadratic order in the fields,

$$
\mathcal{F}=h^{+} M h+\bar{h}^{+} \bar{M} \bar{h}+\mathcal{O}\left((h, \bar{h})^{4}\right),
$$

where

$$
\begin{aligned}
& h=\left(\begin{array}{c}
\alpha \\
v \\
\lambda
\end{array}\right), \quad M=\left(\begin{array}{ccc}
-\frac{1}{8}-\frac{1}{8} C_{0} y^{2} I_{\mathrm{pf}}^{(W)} & \frac{1}{2} & \frac{1}{4} C_{0} y^{2} I_{\mathrm{pf}}^{(W)} \\
\frac{1}{2} & -2 d k & 0 \\
\frac{1}{4} C_{0} y^{2} I_{\mathrm{pf}}^{(W)} & 0 & \frac{1}{2}-\frac{1}{2} C_{0} y^{2} I_{\mathrm{pf}}^{(W)}
\end{array}\right), \\
& \bar{h}=\left(\begin{array}{c}
\bar{\alpha} \\
\bar{v} \\
\bar{\lambda}
\end{array}\right), \quad \bar{M}=\left(\begin{array}{ccc}
-\frac{1}{8}+\frac{1}{8} C_{0} y^{2} I_{\mathrm{pa}}^{(W)} & \frac{1}{2} & -\frac{1}{4} C_{0} y^{2} I_{\mathrm{pa}}^{(W)} \\
\frac{1}{2} & 2 d k & 0 \\
-\frac{1}{4} C_{0} y^{2} I_{\mathrm{pa}}^{(W)} & 0 & \frac{1}{2}+\frac{1}{2} C_{0} y^{2} I_{\mathrm{pa}}^{(W)}
\end{array}\right),
\end{aligned}
$$

with

$$
I_{\mathrm{pf}}^{(W)}=\int_{-\pi}^{\pi} \frac{\mathrm{d}^{d} p}{(2 \pi)^{d}} \frac{F^{4}(p)}{s^{2}(p)}, \quad I_{\mathrm{pa}}^{(W)}=\int_{-\pi}^{\pi} \frac{\mathrm{d}^{d} p}{(2 \pi)^{d}} \frac{F^{2}(p) F_{\pi}^{2}(p)}{s^{2}(p)}
$$

From Eqs. (A.4,A.5) one finds the curves

$$
\begin{aligned}
& k_{\mathrm{pf}}^{(W)}(y)=\frac{1}{d}\left(1-\frac{2^{[d / 2]}}{2} n_{f} y^{2} I_{\mathrm{pf}}^{(W)}\right), \\
& k_{\mathrm{pa}}^{(W)}(y)=\frac{1}{d}\left(-1-\frac{2^{[d / 2]}}{2} n_{f} y^{2} I_{\mathrm{pa}}^{(W)}\right),
\end{aligned}
$$


as second order transition lines for the PM-FM and PM-AM transitions in the small- $y$ region, respectively. For $d=4$ they coincide with the formulas (33) in the main text, and for $y=0$ one recovers the phase structure of the $\mathrm{O}(4)$ model.

The region of strong Yukawa coupling $y$ can be studied similarly, with the integral $I$ of Eq. (A.2) replaced by its large- $y$ analogue (see Ref. [27]). One finds PM, FM and AM phases separated by the lines

$$
\begin{aligned}
& k_{\mathrm{pf}}^{(S)}(y)=\frac{1}{d}\left(1-\frac{2^{[d / 2]}}{2} n_{f} \frac{1}{y^{2}} I_{\mathrm{pf}}^{(S)}\right), \\
& k_{\mathrm{pa}}^{(S)}(y)=\frac{1}{d}\left(-1-\frac{2^{[d / 2]}}{2} n_{f} \frac{1}{y^{2}} I_{\mathrm{pa}}^{(S)}\right),
\end{aligned}
$$

with

$$
I_{\mathrm{pf}}^{(S)}=\int_{-\pi}^{\pi} \frac{\mathrm{d}^{d} p}{(2 \pi)^{d}} \frac{s^{2}(p)}{F^{4}(p)}, \quad I_{\mathrm{pa}}^{(S)}=\int_{-\pi}^{\pi} \frac{\mathrm{d}^{d} p}{(2 \pi)^{d}} \frac{s^{2}(p)}{F^{2}(p) F_{\pi}^{2}(p)},
$$

provided these integrals converge. For the Roma form factor (28) they do converge in $d=4$ dimensions. For the local Zaragoza form factor (雨), however, they are divergent for $d=4$, suggesting that large-y PM and AM phases are absent in this model [27. In this case there are some subtle aspects to the phase structure in the limit $y \rightarrow \infty$, however, which will be discussed separately in Appendix B.

\section{B Appendix}

As we have seen, the mean field determination of the phase structure of the $\mathrm{SU}(2) \times \mathrm{SU}(2)$ chiral Yukawa model with the local form factor (4)

$$
F(p)=\prod_{\mu=1}^{d} \cos \left(p_{\mu} / 2\right)
$$

in the strong Yukawa coupling region, is complicated by the divergence of certain integrals (A.12). It was concluded in Ref. [27] that there are no PM and AM phases at large $y$, in contrast with the $\mathrm{O}(4)$ model to which the model should reduce in the limit $y \rightarrow \infty$, cf. the discussion in Sect. 4. Here we analyse this issue carefully and resolve the paradox. 
First we investigate, in the large- $y$ region along the line $\kappa=0$, whether or not there is a FM solution to the saddle-point equations with lower free energy than the PM candidate solution given by $\Lambda=v=\alpha=0$. As we will see, the answer depends crucially on the form factor $F$. For the local form factor $(B .1)$, there turns out to be a FM solution which is favoured relative to the PM solution for all large but finite $y$, as suggested by the (minus) infinite coefficient of the $1 / y^{2}$ terms in Eq. (A.11).

We start from Eq. (A.1) for the mean field free energy $\mathcal{F}$ and take the staggered fields equal to zero. The saddle-point equations to be satisfied by the mean fields are

$$
\begin{aligned}
\alpha & =4 d k v \\
v & =\dot{u}+\Lambda+\frac{\Lambda^{2} \dddot{u}}{2 \ddot{u}^{2}} \\
\Lambda & =4 C_{0} \ddot{u} \int_{-\pi}^{\pi} \frac{d^{d} p}{(2 \pi)^{d}} \frac{(\dot{u}+\Lambda) s^{2}}{(\dot{u}+\Lambda)^{2} s^{2}+y^{2} F^{4}},
\end{aligned}
$$

where $\Lambda=-\lambda \sqrt{\ddot{u}(\alpha)}$, and $C_{0}=2^{[d / 2]} n_{f} / 2$.

For $k=0$,

$$
\mathcal{F}=2 \Lambda^{2}-2 C_{0} \int_{-\pi}^{\pi} \frac{d^{d} p}{(2 \pi)^{d}} \log \frac{\Lambda^{2} s^{2}+y^{2} F^{4}}{y^{2} F^{4}}
$$

and Eq. (B.2) simplifies to

$$
\begin{aligned}
\alpha & =0 \\
v & =\Lambda=-\frac{1}{2} \lambda, \\
1 & =C_{0} \frac{1}{y^{2}} I_{0}(v / y),
\end{aligned}
$$

where the function

$$
I_{0}(x)=\int_{-\pi}^{\pi} \frac{d^{d} p}{(2 \pi)^{d}} \frac{s^{2}}{x^{2} s^{2}+F^{4}}
$$

is seen to increase with decreasing $x$. 
For not too large $y$, these equations (B.4) have a non-trivial solution, and using the inequality

$$
\forall q>0: \quad \frac{q}{q+1}<\ln (1+q)
$$

with

$$
q=\frac{\Lambda^{2} s^{2}}{y^{2} F^{4}}
$$

one can verify that any such solution has lower free energy $\mathcal{F}$ than the competing PM solution. This holds for both the Roma form factor $F_{R}(28)$ and the local form factor (B.1).

Whether there is a FM solution for arbitrarily large $y$ depends on the small$x$ behaviour of the function (B.5). If it does not blow up for $x \rightarrow 0$, i.e., if $I_{0}(0)$ is a convergent integral, then $I_{0}(v / y)$ is bounded from above by $I_{0}(0)$ and the last equation of (B.4) admits no non-trivial solution for large enough $y$, implying a transition to a PM phase at a finite value of $y$. This happens for the Roma form factor $F_{R}(28)$ in four dimensions.

For the local form factor $(\overline{\mathrm{B} .1})$, however, the fact that $I_{0}(x)$ blows up for $x \rightarrow 0$ means that for all $y<\infty$ there is a FM solution $v$ to this equation. In fact, we will demonstrate that

$$
I_{0}(x) \sim x^{-3 / 2}\left(\log \frac{1}{x}\right)^{d-2} \quad(x \rightarrow 0)
$$

in dimensions $d \geq 2$ (and $\sim x^{-1}$ for $d=1$ ). This implies that the FM solution to Eq. (B.4) behaves like

$$
v=\Lambda \sim\left(\frac{1}{y}\right)^{1 / 3}(\log y)^{(2 / 3)(d-2)} \quad(y \rightarrow \infty)
$$

(we shall assume $d \geq 2$ from now on), indicating an approach to a PM phase with a second order transition at $y=\infty$, with critical exponents given by (B.9).

This argument can be generalized for the approach of the $y \rightarrow \infty$ limit along any line of constant $k$ satisfying $-k_{c} \leq k \leq k_{c}$, where $k_{c}=1 / d$ is the $\mathrm{O}(4)$ critical point. Since we are close to a PM phase, we can expand Eq. (B.2. $)$ to lowest order in the mean fields, provided $k_{c}-k$ is not too small. Using again the small- $x$ behaviour (B.8) of $I_{0}(x)$, we find that 


$$
\begin{array}{ll}
v \sim\left(\frac{k_{c}}{k_{c}-k}\right)^{2 / 3}\left(\frac{1}{y}\right)^{1 / 3}\left|\log \left(y \sqrt{\frac{k_{c}-k}{k_{c}}}\right)\right|^{(2 / 3)(d-2)}, \\
\alpha=4 \frac{k}{k_{c}} v, & \Lambda \sim\left(\frac{k_{c}-k}{k_{c}}\right) v,
\end{array}
$$

for $y \rightarrow \infty$ along lines of constant $k<k_{c}$. The width of the small-(1/y) region where this approximation is valid shrinks to zero for $k \rightarrow k_{c}$, and for $k=k_{c}$ we find instead:

$$
\begin{aligned}
& v \sim \alpha \sim\left(\frac{1}{y}\right)^{1 / 7}(\log y)^{(2 / 7)(d-2)} \\
& \Lambda \sim v^{3} \sim\left(\frac{1}{y}\right)^{3 / 7}(\log y)^{(6 / 7)(d-2)}
\end{aligned}
$$

When the point $\left(y=\infty, k=k_{c}\right)$ is approached from below along curves of the form $1 / y \sim\left(\left(k_{c}-k\right) / k_{c}\right)^{\beta}(\beta>0)$, the mean fields go like

$$
\begin{aligned}
& v \sim \alpha \sim\left(k_{c}-k\right)^{(\beta-2) / 3}, \quad \Lambda \sim\left(k_{c}-k\right)^{(\beta+1) / 3} \quad(\beta \geq 7 / 2), \\
& v \sim \alpha \sim\left(k_{c}-k\right)^{\beta / 7}, \quad \Lambda \sim\left(k_{c}-k\right)^{3 \beta / 7} \quad(0 \leq \beta \leq 7 / 2)
\end{aligned}
$$

up to logarithmic corrections. In the limiting case $\beta \rightarrow \infty$, corresponding to approaching the point from below on the $y=\infty$ axis, one finds $v \sim \alpha \sim \Lambda \sim 0$, as expected for the $\mathrm{O}(4)$ model in the PM phase.

We see that in this scaling region the fermion mass $m_{f}^{2} \sim y^{2} / v^{2}$ blows up when the phase transition is approached, so that the fermions do not survive the continuum limit.

For $k>k_{c}$ one finds in the limit $y \rightarrow \infty$ along lines of constant $k$, for $k-k_{c}$ not too small:

$$
\begin{aligned}
& \alpha \sim\left(\frac{k-k_{c}}{k_{c}}\right)^{1 / 2}, \quad v=\frac{k_{c}}{k} \frac{\alpha}{4}, \\
& \Lambda \sim\left(\frac{k-k_{c}}{k_{c}}\right)^{-1 / 4}\left(\frac{1}{y}\right)^{1 / 2}\left|\log \left(y \sqrt{\frac{k_{c}}{k-k_{c}}}\right)\right|^{d-2},
\end{aligned}
$$

which reproduces the mean field results for the FM phase of the $\mathrm{O}(4)$ model for $y=\infty$.

Note finally that in all cases considered here the asymptotic behaviour of the product of mean fields $\alpha \Lambda^{2}$ or $v \Lambda^{2}$ is $\sim(1 / y)|\log (y / v)|^{2(d-2)}$, as can also 
be deduced directly from Eq. (B.2).

In conclusion, we see that, for the local form factor (四), the vertical line element $y=\infty, k \in\left[-k_{c}, k_{c}\right]$ is a second order phase transition line connecting the large- $y$ FM phase in the region $y<\infty$ to the PM phase of the $\mathrm{O}(4)$ model at $y=\infty$. This result also implies that the divergence of the integral $I_{\mathrm{pa}}^{(S)}$ (A.12) becomes meaningless, because the transition line $k_{p a}^{(S)}(y)$ between PM and AM phases has shrunk to a single point $B=\left(y=\infty, k=-k_{c}\right)$ on the $y=\infty$ axis.

The promised demonstration concerning the $x \rightarrow 0$ behaviour $(\mathbb{B} .8)$ of the integral $I_{0}(x)$ for the local form factor $(\mathbb{B} .1)$ is as follows. We shall consider the function

$$
I_{1}(x)=x^{2} I_{0}(x)=\int_{-\pi}^{\pi} \frac{d^{d} p}{(2 \pi)^{d}} \frac{x^{2} s^{2}}{x^{2} s^{2}+F^{4}}=\int_{-\pi}^{\pi} \frac{d^{d} p}{(2 \pi)^{d}} \frac{x^{2} s^{2}}{x^{2} s^{2}+F_{\pi}^{4}} .
$$

For $d=1$ this integral can be computed exactly. One finds

$$
I_{1}(x)=\int_{-\pi}^{\pi} \frac{d p}{2 \pi}\left(1+\frac{\cos ^{2}(p / 2)}{\left(4 x^{2}\right) \sin ^{2}(p / 2)}\right)^{-1}=\frac{2 x}{1+2 x}
$$

For the general case $d \geq 2$ we shall show that, for small enough $x, I_{1}(x)$ satisfies

$$
C_{1} x^{1 / 2}\left(\log \frac{1}{x}\right)^{d-2}<I_{1}(x)<C_{2} x^{1 / 2}\left(\log \frac{1}{x}\right)^{d-2}
$$

for positive constants $C_{1,2}$.

A simple order of magnitude estimate already hints at an $x^{1 / 2}$ behaviour. The dominant contribution to the integral $I_{1}(x)$ for small $x$ comes from the regions where $F^{4} \lesssim x^{2} s^{2}$, such that the integrand is $\mathcal{O}(1)$. The $x$-dependent size of these regions determines how large this contribution is. In this way one finds that the dominant contribution is of order $x^{1 / 2}$ and comes from the regions where $N(1 \leq N \leq d-1)$ of the momentum components $p_{\mu}$ are close to $\pi$, with the other $p_{\mu}$ away from both 0 and $\pi$.

Rigorous bounds on $I_{1}(x)$ are obtained as follows. For the lower bound one has $s^{2}(p) \geq \sin ^{2} p_{1}$, so that

$$
I_{1}(x) \geq \int_{-\pi}^{\pi} \frac{d^{d} p}{(2 \pi)^{d}}\left(1+\frac{\cos ^{2}\left(p_{1} / 2\right)}{\left(4 x^{2}\right) \sin ^{2}\left(p_{1} / 2\right)} \prod_{\mu=2}^{d} \sin ^{4}\left(p_{\mu} / 2\right)\right)^{-1} .
$$


The integration over $p_{1}$ can be done using the $d=1$ result $(\mathbb{B} .19)$, and subsequently the $p_{2}$-integration gives

$$
I_{1}(x) \geq \int_{-\pi}^{\pi} \frac{d p_{3}}{2 \pi} \ldots \frac{d p_{d}}{2 \pi} \sqrt{\frac{2 x}{2 x+\sin ^{2}\left(p_{3} / 2\right) \ldots \sin ^{2}\left(p_{d} / 2\right)}}
$$

For the upper bound, we divide the integration region into subregions $R_{j}$ defined such that $p \in R_{j}$ if $\sin ^{2} p_{j} \geq \sin ^{2} p_{k}$ for all $k \neq j: \int_{-\pi}^{\pi} d^{d} p /(2 \pi)^{d}=\sum_{j} \int_{R_{j}}$. On $R_{j}, s^{2} \leq d \sin ^{2} p_{j}$, and using symmetry and positivity of the integrand one obtains

$$
\begin{aligned}
I_{1}(x) & \leq \sum_{j} \int_{R_{j}}\left(1+\frac{\cos ^{2}\left(p_{j} / 2\right)}{\left(4 d x^{2}\right) \sin ^{2}\left(p_{j} / 2\right)} \prod_{\mu \neq j} \sin ^{4}\left(p_{\mu} / 2\right)\right)^{-1} \\
& \leq d \int_{-\pi}^{\pi} \frac{d^{d} p}{(2 \pi)^{d}}\left(1+\frac{\cos ^{2}\left(p_{1} / 2\right)}{\left(4 d x^{2}\right) \sin ^{2}\left(p_{1} / 2\right)} \prod_{\mu=2}^{d} \sin ^{4}\left(p_{\mu} / 2\right)\right)^{-1}
\end{aligned}
$$

leading to an expression similar to (B.22).

Using furthermore that $\left(p_{k} / \pi\right)^{2} \leq \sin ^{2}\left(p_{k} / 2\right) \leq\left(p_{k} / 2\right)^{2}$, we get

$$
G\left[x\left(\frac{2}{\pi}\right)^{2(d-2)}\right] \leq I_{1}(x) \leq d G[x \sqrt{d}]
$$

where

$$
G[x] \equiv \int_{0}^{1} d k_{3} \ldots \int_{0}^{1} d k_{d} \sqrt{\frac{2 x}{2 x+k_{3}^{2} \ldots k_{d}^{2}}}
$$

We see that the limiting behaviour of $I_{1}(x)$ is determined by that of $G[x]$.

In order to obtain an upperbound on $G[x]$ we divide the integration region into $2^{d}$ subregions $S_{i}$ by writing

$$
\int_{0}^{1} d k_{\mu}=\int_{0}^{\sqrt{2 x}} d k_{\mu}+\int_{\sqrt{2 x}}^{1} d k_{\mu} \quad(\mu=3, \ldots, d) .
$$


On those regions $S_{i}$ where one or more of the $k_{\mu}$ are smaller then $\sqrt{2 x}$, we write

$$
I_{S_{i}}=\int_{S_{i}} \sqrt{\frac{2 x}{2 x+k_{3}^{2} \ldots k_{d}^{2}}} \leq \int_{S_{i}} 1 \leq \sqrt{2 x} .
$$

The remaining contribution is

$$
\begin{aligned}
& \int_{\sqrt{2 x}}^{1} d k_{3} \ldots \int_{\sqrt{2 x}}^{1} d k_{d} \sqrt{\frac{2 x}{2 x+k_{3}^{2} \ldots k_{d}^{2}}} \\
& \quad \leq \int_{\sqrt{2 x}}^{1} d k_{3} \ldots \int_{\sqrt{2 x}}^{1} d k_{d} \frac{\sqrt{2 x}}{k_{3} \ldots k_{d}}=\sqrt{2 x}\left(\frac{1}{2} \log \frac{1}{x}\right)^{d-2}
\end{aligned}
$$

To obtain a lower bound on $G[x]$, we write

$$
G[x] \geq \int_{(2 x)^{\beta}}^{1} d k_{3} \ldots \int_{(2 x)^{\beta}}^{1} d k_{d} \sqrt{\frac{2 x}{2 x+k_{3}^{2} \ldots k_{d}^{2}}}
$$

guided by what we have seen is a momentum region providing a large contribution to the integral. Taking $\beta=1 /(2 d-4)$, we get

$$
G[x] \geq \int_{(2 x)^{\beta}}^{1} d k_{3} \ldots \int_{(2 x)^{\beta}}^{1} d k_{d} \frac{\sqrt{x}}{k_{3} \ldots k_{d}}=\sqrt{x}\left(\frac{1}{2(d-2)} \log \frac{1}{x}\right)^{d-2}
$$

Combining the bounds $(\mathrm{B} .28, \mathrm{~B} .30)$ on $G[x]$ with the estimate (B.24) for $I_{1}(x)$ we finally arrive at the desired result $(\mathrm{B} .20)$ with

$$
C_{1}=\left(\frac{1}{\pi(d-2)}\right)^{d-2}, \quad C_{2}=\sqrt{2} d^{5 / 4}\left(\frac{1}{2}\right)^{d-2} .
$$

For $d=2$ it is not difficult to improve the lower bound to $C_{1}=\sqrt{2}$ while for $d=3$ stricter estimates lead to the slightly stronger bounds $C_{1}=\sqrt{2} / \pi$, $C_{2}=3^{5 / 4} \sqrt{2} / \pi$.

\section{References}


[1] L.H. Karsten and J. Smit, Nucl. Phys. B183 (1981) 103; H.B. Nielsen and M. Ninomiya, Nucl. Phys. B185 (1981) 20; B193 (1981) 173.

[2] D.N. Petcher, Nucl. Phys. B (Proc. Suppl.) 30 (1993) 50.

[3] G. Münster, Nucl. Phys. B (Proc. Suppl.) 42 (1995) 162.

[4] J. Smit, Acta Physica Polonica B17 (1986) 531; Nucl. Phys. B175 (1980) 307; P.V.D. Swift, Phys. Lett. B145 (1984) 256.

[5] T. Appelquist and J. Carazzone, Phys. Rev. D11 (1975) 2856.

[6] M. Veltman, Nucl. Phys. B123 (1977) 89.

[7] D. Toussaint, Phys. Rev. D18 (1978) 1628.

[8] M.J. Dugan and L. Randall, Nucl. Phys. B382 (1992) 419.

[9] I. Montvay, Phys. Lett B199 (1987) 89; B205 (1988) 315.

[10] J.L. Alonso, Ph. Boucaud, J.L. Cortés and E. Rivas, Nucl. Phys. B (Proc. Suppl.) 17 (1990) 461.

[11] J.L. Alonso, Ph. Boucaud, J.L. Cortés and E. Rivas, Mod. Phys. Lett. A5 (1990) 275.

[12] J.L. Alonso, Ph. Boucaud, J.L. Cortés and E. Rivas, Phys. Rev. D44 (1991) 3258.

[13] A. Borrelli, L. Maiani, G.C. Rossi, R. Sisto and M. Testa, Nucl. Phys. B333 (1990) 335.

[14] L. Maiani, Nucl. Phys. B (Proc. Suppl.) 29B,C (1992) 33.

[15] M.F.L. Golterman and D.N. Petcher, Phys. Lett. B225 (1989) 159.

[16] J.L. Alonso, Ph. Boucaud, J.L. Cortés, F. Lesmes and E. Rivas, Nucl. Phys. B407 (1993) 373.

[17] L. Maiani, G.C. Rossi and M. Testa, Phys. Lett. B292 (1992) 397.

[18] J. Smit, Nucl. Phys. B (Proc. Suppl.) 26 (1992) 480; Nucl. Phys. B (Proc. Suppl.) 29B,C (1992) 83; W. Bock, J. Smit and J.C. Vink, Phys. Lett. B291 (1992) 297.

[19] For a review see, e.g., Y. Shamir, in Lattice 95, Proceedings of the International Symposium, eds. T. Kieu et al., Nucl. Phys. B (Proc. Suppl.), to be published.

[20] J.L. Alonso, Ph. Boucaud, F. Lesmes and A.J. van der Sijs, Nucl. Phys. $B$ (Proc. Suppl.) 42 (1995) 595.

[21] W. Bock, A.K. De, C. Frick, K. Jansen and T. Trappenberg, Nucl. Phys. B371 (1992) 683; W. Bock, A.K. De, C. Frick, J. Jersák and T. Trappenberg, Nucl. Phys. B378 (1992) 652; M.F.L. Golterman, D.N. Petcher and E. Rivas, Nucl. Phys. B377 (1992) 405.

[22] C. Frick, L. Lin, I. Montvay, G. Münster, M. Plagge, T. Trappenberg and H. Wittig, Nucl. Phys. B397 (1993) 431.

[23] M.F.L. Golterman, D.N. Petcher and J. Smit, Nucl. Phys. B370 (1992) 51.

[24] A.K. De and J. Jersák, in "Heavy Flavours", eds. A. Buras and M. Lindner (World Scientific, Singapore, 1992).

[25] J.L. Alonso, Ph. Boucaud, F. Lesmes and A.J. van der Sijs, preprint DFTUZ/95/22 (Zaragoza), LPTHE 95/59 (Orsay), hep-lat/9509nnn, to be published in the Proceedings of Lattice 95 (Melbourne, Australia, 11- 
15 July 1995), eds. T. Kieu et al., Nucl. Phys. B (Proc. Suppl.).

[26] J.L. Alonso, Ph. Boucaud and A.J. van der Sijs, in preparation.

[27] J.L. Alonso, Ph. Boucaud, F. Lesmes and E. Rivas, Phys. Lett. B329 (1994) 75 .

[28] J.L. Alonso, Ph. Boucaud, J.L. Cortés, F. Lesmes and E. Rivas, Nucl. Phys. B (Proc. Suppl.) 29B,C (1992) 171.

[29] T. Reisz, Comm. Math. Phys. 116 (1988) 81, 573; 117 (1988) 79, 639.

[30] M. Lüscher, in "Fields, strings and critical phenomena" (Les Houches 1988), eds. E. Brézin and J. Zinn-Justin (North-Holland, Amsterdam, 1990).

[31] J.C. Vink, Phys. Lett. B321 (1994) 239.

[32] J. Smit, Nucl. Phys. B (Proc. Suppl.) 4 (1988) 451.

[33] J. Smit, Nucl. Phys. B (Proc. Suppl.) 17 (1990) 3.

[34] J.L. Alonso, Ph. Boucaud and A.J. van der Sijs, Phys. Rev. D52 (1995) 1732.

[35] S. Tominaga and S.V. Zenkin, Phys. Rev. D50 (1994) 3387.

[36] I.H. Lee, J. Shigemitsu and R.E. Schrock, Nucl. Phys. B330 (1990) 225.

[37] W. Bock, A.K. De, K. Jansen, J. Jersák, T. Neuhaus and J. Smit, Nucl. Phys. B344 (1990) 207.

[38] G. Gallavotti and V. Rivasseau, Phys. Lett. 122B (1983) 268; Ann. Inst. H. Poincaré 40 (1984) 185; D.J.E. Callaway, Phys. Rep. 167 (1988) 241. 